\title{
Imaging of congenital torticollis in infants: a retrospective study of an institutional protocol
}

\author{
Nicholas Boyko, BS, ${ }^{1-3}$ Melissa Ann Eppinger, BA, ${ }^{2,3}$ Deborah Straka-DeMarco, PT, ${ }^{4}$ and \\ Catherine Anne Mazzola, MD2-4 \\ ${ }^{1}$ Rowan University School of Osteopathic Medicine, Stratford; ${ }^{2}$ Department of Neuroscience, and ${ }^{4}$ Craniofacial and \\ Plagiocephaly Clinics, Goryeb Children's Hospital, Morristown Medical Center, Morristown; and ${ }^{3}$ New Jersey Pediatric \\ Neuroscience Institute, Morristown, New Jersey
}

\begin{abstract}
OBJECTIVE The authors' objectives in this study were to evaluate their institutional protocol and create recommendations for radiographic imaging in infants with torticollis, with or without plagiocephaly, based on a retrospective clinical analysis and literature review.
\end{abstract}

METHODS A retrospective analysis was conducted to identify infants with torticollis who were evaluated by the craniofacial team. The following patients were identified: those who underwent imaging in the form of radiography, CT scanning, or MRl; those who had a secondary diagnosis or additional clinical finding as the indication for imaging; and those with persistent torticollis despite completion of recommended repositioning techniques and physical therapy (PT).

RESULTS A total of 683 infants between the ages of 1 day and 335 days (mean age 141.6 days) were referred for management of torticollis. Initial imaging was performed in 48 patients, of whom 44 were identified as having a diagnosis other than torticollis or a significant clinical finding as the indication for imaging. The remaining 4 patients $(0.6 \%$ of the torticollis population) underwent imaging and did not have an additional diagnosis or a significant clinical finding. There were no abnormal radiological findings in these patients. Of the 639 patients diagnosed with congenital torticollis who did not have a secondary diagnosis that required initial imaging, 99.2\% (634 patients) achieved resolution of torticollis with recommended repositioning techniques and completion of PT and without radiography, CT, or MRI.

CONCLUSIONS Within the limits of this retrospective study and supporting literature, the authors provide the following recommendations regarding radiographic imaging of infants with congenital torticollis. 1) Clinical examination is sufficient in the diagnosis of congenital torticollis in infants. Radiography, CT scanning, and MRI are not necessary. 2) In cases in which additional clinical or neurological findings are present, imaging may be recommended. 3) PT and repositioning techniques are the recommended treatment modalities for congenital torticollis. 4) Additional evaluations should be performed throughout the duration of PT to assess progress and response to therapy. 5) Imaging should be performed to rule out nonmuscular pathologies if torticollis persists after 1 year of treatment.

https://thejns.org/doi/abs/10.3171/2017.3.PEDS16277

KEY WORDS congenital; infant; pediatric; plagiocephaly; recommendations; torticollis; functional neurosurgery

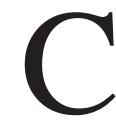
ONGENITAL torticollis presents with lateral neck flexion and neck rotation to the opposite side, as shown in Fig. 1. The incidence of torticollis has been reported to be as high as $8.2 \%-16 \%, 9,15$ but it is more commonly reported as between $0.3 \%$ and $3.92 \% ., 4,7,8,14,16$ The cause of congenital torticollis may be due to an abnormal position of the intrauterine fetus or fetal head descent causing trauma to the sternocleidomastoid muscle or its innervation. ${ }^{79}$ Birth trauma may result in compartment syndrome, which is characterized by ischemia and edema of the sternocleidomastoid muscle; biopsy studies have shown that fibrous tissue replaces healthy muscle. ${ }^{9}$ Torticollis is a risk factor for positional plagiocephaly, an acquired deformation of the skull. More than $90 \%$ of infants with positional plagiocephaly have torticollis that is likely attributed to the positional preference of the neck, resulting in repeated pressure to the skull while supine.?

If left untreated, torticollis may lead to skull base and

ABBREVIATIONS EMR = electronic medical record; PT = physical therapy.

SUBMITTED May 18, 2016. ACCEPTED March 28, 2017.

INCLUDE WHEN CITING Published online June 2, 2017; DOI: 10.3171/2017.3.PEDS16277. 
facial asymmetry, requiring surgical correction. ${ }^{5,8,9,14,16}$ Torticollis is also associated with scoliosis, hip dysplasia, brachial plexus injury, distal extremity deformity, early and persistent developmental delays, and temporomandibular joint dysfunction. ${ }^{8,9,13}$ Deformations begin in infancy; therefore, early identification and treatment is critical. ${ }^{17}$ In the absence of an unrelated significant clinical finding, congenital torticollis is successfully treated with repositioning techniques and early physical therapy (PT) ${ }^{8,16}$ It has been suggested in previous reports that radiography, CT, and MRI are often unnecessary, as bony abnormalities are rarely found in conjunction with torticollis. Snyder and Coley ${ }^{14}$ published a retrospective study in which they analyzed radiographic images obtained in 502 infants with nontraumatic torticollis. Their results revealed that 4 of 502 patients had abnormal findings, and they found only 1 patient with an abnormality contraindicating management with PT. The authors concluded that radiographic imaging should only be recommended for patients with abnormal clinical findings or persistent torticollis after PT treatment. ${ }^{14}$ Yet physicians who first evaluate a patient with suspected congenital torticollis may order imaging prior to referral to a specialist.

The Goryeb Children's Hospital Craniofacial Team clinical protocol includes examination of infants by the craniofacial team. This team comprises a pediatric neurological surgeon, a developmental pediatrician, and a pediatric physical therapist. The head and neck are examined for postural abnormalities, and a neurodevelopmental examination is performed. Infants with uncomplicated torticollis are eligible for management with repositioning techniques and PT without imaging. Imaging is recommended for infants with a clinical finding suggestive of a secondary condition or nonmuscular etiology for torticollis.

The PT treatment protocol consists of one 60-minute session per week. Stretching and flexibility exercises are recommended to improve range of motion, balance, coordination, and posture. Caregivers are encouraged to reposition infants, while awake, into the supine position throughout the day to promote strength development.

Reassessment of torticollis and response to therapy is performed every 2 to 3 months. PT is continued until torticollis resolves. Resolution is defined as full range of motion in the cervical spine and the ability to maintain the head in the midline position. If torticollis persists after 12 months despite therapy, diagnostic imaging is performed.

\section{Methods}

As part of a quality improvement initiative, a General Electric Centricity database was searched for all patients with a diagnosis of torticollis who were evaluated between the period of August 5, 2005, and July 23, 2015. Data were reviewed by the research team. A search was performed to identify all medical records of patients with a diagnosis of torticollis. Patients older than 12 months of age at the initial visit and those lost to follow-up of their condition were removed from the sample population. Clinical data reviewed in each of the electronic charts included coexisting diagnoses, modalities of imaging used (radiography, $\mathrm{CT}$, or MRI), and indication for imaging and correspond-

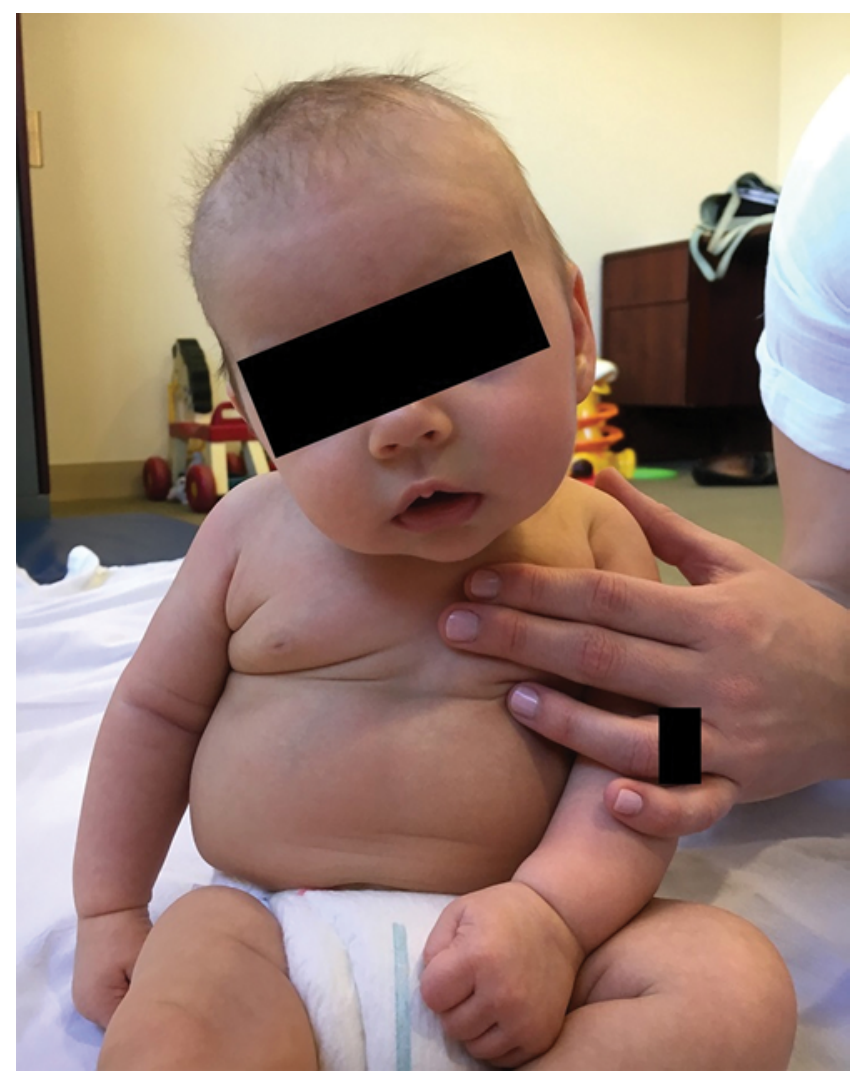

FIG. 1. Photograph of an infant with congenital torticollis. Note the lateral flexion and rotation to the opposite side. Positional plagiocephaly is noted by the anterior displacement of the left ear and facial structures. Torticollis and plagiocephaly resolved in this patient after repositioning techniques and PT. Figure is available in color online only.

ing interpretation. Patients whose torticollis resolved with PT and repositioning techniques were identified, along with those who remained symptomatic after 1 year.

\section{Results}

A total of 742 infants were evaluated for torticollis. Of these patients, 59 were excluded from the sample population: 57 patients were older than 12 months of age at the initial visit and 2 had undergone management of torticollis at an outside institution. The remaining 683 patients were included in the study and ranged in age from 1 day to 335 days (mean age 141.6 days) on the day of the initial visit. Forty-eight infants (7.0\% of the patient population) underwent initial imaging. Forty-four patients (6.4\%) affected by torticollis were identified as having a concomitant diagnosis or significant clinical finding as the indication for image request, while the remaining 4 patients $(0.6 \%)$ lacked further indication to support imaging (Fig. 2). Indications for radiographic imaging included clinical findings discovered by the craniofacial team in addition to findings noted on previous imaging studies ordered by the referring physician. The most common additional findings were macrocephaly, ridging of sutures noted on physical examination, obstructive hydrocephalus, tethered spinal cord or other congenital anomaly of the spinal cord, and spastic hemiparesis. A complete list of indications for the utilization of 


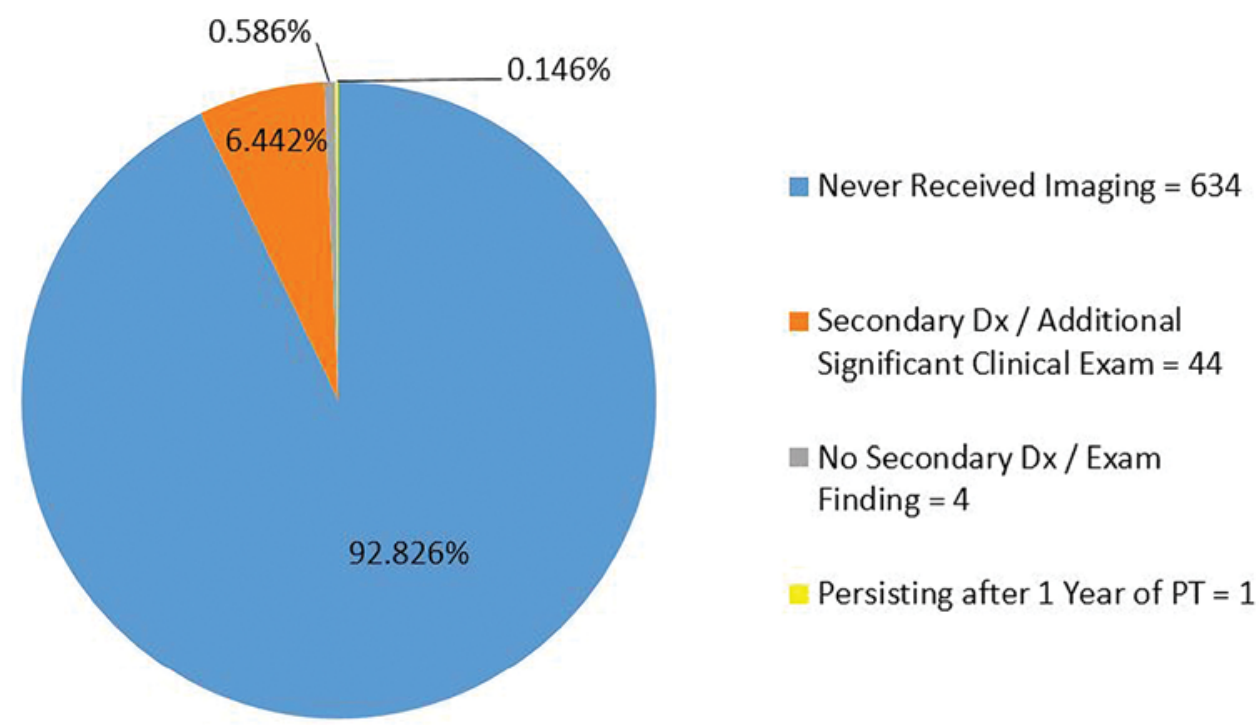

FIG. 2. Pie chart showing the proportions of New Jersey Pediatric Neuroscience Institute's 683 infantile torticollis patients. Dx = diagnosis. Figure is available in color online only.

radiographic imaging and the number of patients identified with each are referenced in Table 1.

Six of the 44 patients were identified with ridging of skull sutures noted on physical examination, and imaging was subsequently performed. CT scanning was performed in 3 patients, radiography in 2 patients, and radiography and then CT scanning the following week in 1 patient. Of the patients who underwent CT scanning, one patient underwent CT scanning of the head and brain without contrast and was subsequently diagnosed with craniosynostosis. Another patient underwent CT scanning of the head, which revealed a $1.5-\mathrm{cm}$ thyroglossal duct cyst and no craniosynostosis. The remaining patient underwent $\mathrm{CT}$ scanning of the head and brain without contrast, which revealed right parietal plagiocephaly, right frontal bossing, and no brain abnormalities or craniosynostosis. Complete skull radiographs were obtained in both patients who underwent radiography, which revealed craniosynostosis. The sixth patient underwent radiography of the skull, and interpretation of the findings was inconclusive. Craniosynostosis was ruled out with reconstructive $\mathrm{CT}$ of the skull obtained 7 days later. Overall, 3 of the 6 patients were diagnosed with craniosynostosis following imaging.

The remaining 4 of the 48 patients $(0.6 \%$ of the torticollis population) underwent initial imaging and did not have an additional diagnosis or significant clinical finding as an indication for imaging. Two of the patients underwent CT scanning of the head and brain without contrast, one underwent radiography of the neck, and the remaining patient underwent complete skull radiography. Abnormal radiological findings were not identified in these patients.

One patient $(0.2 \%)$ did not undergo initial imaging or achieve resolution of torticollis after 1 year of treatment with recommended repositioning techniques and PT. Imaging was performed at this time, and an occipital condyle-C1 vertebral fusion abnormality was discovered.

Overall, $92.8 \%$ of our infantile torticollis patient population achieved resolution of torticollis with recommended repositioning techniques and completion of PT without undergoing radiography, CT scanning, or MRI. Excluding the 44 patients with a secondary diagnosis requiring initial imaging from the study's total population $(n=683)$ revealed that $99.2 \%$ (634 of 639) of our patients with uncomplicated torticollis were successfully treated without imaging.

\section{Discussion}

The vast majority (99.2\%) of infants with torticollis were successfully treated without undergoing imaging. The remaining previously mentioned 5 patients included 4 who underwent imaging without an additional indication and 1 who underwent imaging after management with PT had failed. On evaluation of the patients' medical records, radiographic imaging was unnecessary in 4 of the patients and should not have been performed. Only 1 patient did not undergo initial imaging or achieve resolution of torticollis after 1 year of repositioning techniques and PT, which represents a small portion of the sample population. This patient's outcome is unlikely to have been improved with earlier imaging. Therefore, our findings, along with those of others, ${ }^{8,14,16}$ favorably demonstrate the treatment and management of uncomplicated congenital torticollis in infants without the utilization of initial radiographic imaging.

This retrospective study has limitations in need of mention. A prospective study of infants treated with and without imaging would provide more valuable data, but it requires a group of infants to receive radiation exposure. We believe that this should be avoided. Also, our screening criteria for radiographic imaging state that we do not recommend imaging unless an additional significant finding is identified. Our protocol uses a multidisciplinary approach to minimize the risk of a missed clinical diagnosis. In addition, our results rely on the accuracy of the electronic medical record (EMR) system. Diagnosis-based searches have been performed successfully using the General Electric Centricity EMR system in other publications on con- 
TABLE 1. Indications for radiographic imaging in 44 patients with torticollis

\begin{tabular}{|c|c|}
\hline Secondary Diagnosis/Clinical Finding & No. of Patients* \\
\hline Macrocephaly & 8 \\
\hline Ridging of sutures noted on physical exam & 6 \\
\hline Obstructive hydrocephalus & 5 \\
\hline $\begin{array}{l}\text { Tethered spinal cord/other congenital anomaly of } \\
\text { spinal cord }\end{array}$ & 4 \\
\hline Spastic hemiparesis & 4 \\
\hline Strabismus & 3 \\
\hline Hypotonia & 3 \\
\hline Communicating hydrocephalus & 3 \\
\hline Delayed development & 2 \\
\hline Closed head injury & 2 \\
\hline Microcephaly & 2 \\
\hline Fracture of vault of skull & 2 \\
\hline Other specified myoneural disorders & 2 \\
\hline Scoliosis associated w/ other condition & 1 \\
\hline Concussion & 1 \\
\hline Subdural hematoma, trauma w/ LOC & 1 \\
\hline Congenital hydrocephalus & 1 \\
\hline Talipes equinovarus, bilateral & 1 \\
\hline Spina bifida occulta & 1 \\
\hline $\begin{array}{l}\text { Closed Fx of base of skull w/ subdural hemorrhage } \\
\text { w/ no LOC }\end{array}$ & 1 \\
\hline Cerebral palsy, infantile & 1 \\
\hline Contusion, scalp & 1 \\
\hline Birth trauma, other injuries to scalp & 1 \\
\hline Hemivertebra & 1 \\
\hline Cervical rib & 1 \\
\hline Klippel-Feil syndrome & 1 \\
\hline Speech or language disorder & 1 \\
\hline Total no. of diagnoses & 60 \\
\hline
\end{tabular}

$\mathrm{FX}=$ fracture; $\mathrm{LOC}=$ loss of consciousness.

* Multiple diagnoses were found in some patients.

ditions unrelated to torticollis. ${ }^{10,11}$ To minimize variability in the EMR review, a single member of our research team performed all data extraction and reviewed all charts with the supervision of the senior author.

Our data suggest that infants with torticollis may be safely managed and treated without imaging, thereby reducing radiation exposure. While radiographs expose the child to minimal radiation, radiation has been postulated to be the cause of some cancers in children. ${ }^{2,3}$ Brenner and Hall demonstrated that infants have the greatest estimated organ dose of radiation per CT study and, therefore, have the greatest risk of developing cancer later in life. ${ }^{2} \mathrm{~A}$ direct correlation existed for patients who had undergone 2 or 3 CT studies, equivalent to $30-90 \mathrm{mSv}$ of radiation, and increased risk of cancer. ${ }^{2}$ The risk of radiation attributed to simple radiographs is much less. Berrington de González and Darby reported that diagnostic radiographs are estimated to account for $0.6 \%-3 \%$ of the cumulative risk of cancer in the developed world. ${ }^{1} \mathrm{CT}$ scans and radiographs are unnecessary in cases of uncomplicated torticollis in infants. Haque et al. as well as Lin and Chou have suggested ultrasonography for evaluation of torticollis. ${ }^{6,12}$

For infants with simple torticollis, PT and treatment without imaging is recommended. Nonmuscular causes of congenital torticollis may include Klippel-Feil syndrome, syringomyelia, occipitoatlantal fusion, spondyloepiphyseal dysplasia, hemivertebra, and subluxation. ${ }^{9}{ }^{914}$ These rare etiologies may require CT scanning and/or MRI for final diagnosis.

\section{Conclusions}

Our retrospective analysis provides Class III evidence for a Level III recommendation regarding the diagnosis, clinical assessment, and management of torticollis in infants. Our recommendations for imaging are as follows. 1) Clinical examination is sufficient in the diagnosis of congenital torticollis in infants. Imaging with radiographs, $\mathrm{CT}$, and MRI is not necessary. 2) In cases in which additional clinical or neurological findings are present, imaging may be recommended. 3) PT and repositioning techniques are the recommended treatment modalities for congenital torticollis. 4) Additional evaluations should be performed throughout the duration of PT to assess progress and response to therapy. 5) Imaging should be performed to rule out nonmuscular pathologies if torticollis persists after 1 year of treatment. It is our hope that these recommendations will further improve the management of infants with congenital torticollis.

\section{Acknowledgments}

We would like to thank Thomas J. Sernas, MS, PA-C, for assisting in the medical care of these patients and for his editorial assistance in preparing this manuscript.

These guidelines were funded exclusively by the New Jersey Pediatric Neuroscience Institute, which received no funding from outside commercial sources to support the development of this study.

\section{References}

1. Berrington de González A, Darby S: Risk of cancer from diagnostic X-rays: estimates for the UK and 14 other countries. Lancet 363:345-351, 2004

2. Brenner DJ, Hall EJ: Computed tomography-an increasing source of radiation exposure. N Engl J Med 357:2277-2284, 2007

3. Chen JX, Kachniarz B, Gilani S, Shin JJ: Risk of malignancy associated with head and neck CT in children: a systematic review. Otolaryngol Head Neck Surg 151:554-566, 2014

4. Chen MM, Chang HC, Hsieh CF, Yen MF, Chen THH: Predictive model for congenital muscular torticollis: analysis of 1021 infants with sonography. Arch Phys Med Rehabil 86:2199-2203, 2005

5. Cheng JC, Wong MW, Tang SP, Chen TM, Shum SL, Wong EM: Clinical determinants of the outcome of manual stretching in the treatment of congenital muscular torticollis in infants. A prospective study of eight hundred and twenty-one cases. J Bone Joint Surg Am 83-A:679-687, 2001

6. Haque S, Bilal Shafi BB, Kaleem M: Imaging of torticollis in children. Radiographics 32:557-571, 2012

7. Hsieh YY, Tsai FJ, Lin CC, Chang FCC, Tsai CH: Breech deformation complex in neonates. J Reprod Med 45:933-935, 2000

8. Kaplan SL, Coulter C, Fetters L: Physical therapy manage- 
ment of congenital muscular torticollis: an evidence-based clinical practice guideline: from the Section on Pediatrics of the American Physical Therapy Association. Pediatr Phys Ther 25:348-394, 2013

9. Kuo AA, Tritasavit S, Graham JM Jr: Congenital muscular torticollis and positional plagiocephaly. Pediatr Rev 35:7987, 2014

10. Levin P, Wei W, Miao R, Ye F, Xie L, Baser O, et al: Therapeutically interchangeable? A study of real-world outcomes associated with switching basal insulin analogues among US patients with type 2 diabetes mellitus using electronic medical records data. Diabetes Obes Metab 17:245-253, 2015

11. Marrett E, Zhao C, Zhang NJ, Zhang Q, Ramey DR, Tomassini JE, et al: Limitations of real-world treatment with atorvastatin monotherapy for lowering LDL-C in high-risk cardiovascular patients in the US. Vasc Health Risk Manag 10:237-246, 2014

12. Lin JN, Chou ML: Ultrasonographic study of the sternocleidomastoid muscle in the management of congenital muscular torticollis. J Pediatr Surg 32:1648-1651, 1997

13. Öhman A, Nilsson S, Lagerkvist AL, Beckung E: Are infants with torticollis at risk of a delay in early motor milestones compared with a control group of healthy infants? Dev Med Child Neurol 51:545-550, 2009

14. Snyder EM, Coley BD: Limited value of plain radiographs in infant torticollis. Pediatrics 118:e1779-e1784, 2006

15. Stellwagen L, Hubbard E, Chambers C, Jones KL: Torticollis, facial asymmetry and plagiocephaly in normal newborns. Arch Dis Child 93:827-831, 2008

16. Ta JH, Krishnan M: Management of congenital muscular torticollis in a child: a case report and review. Int J Pediatr Otorhinolaryngol 76:1543-1546, 2012

17. Yu CC, Wong FH, Lo LJ, Chen YR: Craniofacial deformity in patients with uncorrected congenital muscular torticollis: an assessment from three-dimensional computed tomography imaging. Plast Reconstr Surg 113:24-33, 2004

\section{Disclosures}

The authors report no conflict of interest concerning the materials or methods used in this study or the findings specified in this paper.

\section{Author Contributions}

Conception and design: Straka-DeMarco, Mazzola. Acquisition of data: Boyko, Mazzola. Analysis and interpretation of data: Boyko, Mazzola. Drafting the article: all authors. Critically revising the article: all authors. Reviewed submitted version of manuscript: all authors. Approved the final version of the manuscript on behalf of all authors: Boyko. Statistical analysis: Boyko, Mazzola. Administrative/technical/material support: Mazzola. Study supervision: Mazzola.

\section{Supplemental Information \\ Previous Presentations}

This abstract was presented at the Osteopathic Medical Conference \& Exposition, October 17-21, 2015, in Orlando, FL; at the 84th AANS Annual Scientific Meeting, April 30 to May 4, 2016, in Chicago, IL; and the Atlantic Health System Research Day, June 2016 .

\section{Correspondence}

Nicholas Boyko, New Jersey Pediatric Neuroscience Institute, 131 Madison Ave., Morristown, NJ 07960. email: boykon5@ rowan.edu. 